TRANSACTIONS OF THE

AMERICAN MATHEMATICAL SOCIETY

Volume 356, Number 11, Pages 4255-4272

S 0002-9947(04)03628-1

Article electronically published on June 2, 2004

\title{
MORSE INDEX AND UNIQUENESS FOR POSITIVE SOLUTIONS OF RADIAL $p$-LAPLACE EQUATIONS
}

\author{
AMANDINE AFTALION AND FILOMENA PACELLA
}

\begin{abstract}
We study the positive radial solutions of the Dirichlet problem $\Delta_{p} u+f(u)=0$ in $B, u>0$ in $B, u=0$ on $\partial B$, where $\Delta_{p} u=\operatorname{div}\left(|\nabla u|^{p-2} \nabla u\right)$, $p>1$, is the $p$-Laplace operator, $B$ is the unit ball in $\mathbb{R}^{n}$ centered at the origin and $f$ is a $C^{1}$ function. We are able to get results on the spectrum of the linearized operator in a suitable weighted space of radial functions and derive from this information on the Morse index. In particular, we show that positive radial solutions of Mountain Pass type have Morse index 1 in the subspace of radial functions of $W_{0}^{1, p}(B)$. We use this to prove uniqueness and nondegeneracy of positive radial solutions when $f$ is of the type $u^{s}+u^{q}$ and $p \geq 2$.
\end{abstract}

\section{INTRODUCTION}

Let us consider the Dirichlet problem

$$
\Delta_{p} u+f(u)=0 \text { in } B, \quad u>0 \text { in } B, \quad u=0 \text { on } \partial B,
$$

where $B$ is the unit ball in $\mathbb{R}^{n}$ centered at the origin, $n \geq 2, \Delta_{p}$ denotes the $p$-Laplace operator

$$
\Delta_{p} u=\operatorname{div}\left(|\nabla u|^{p-2} \nabla u\right), \quad p>1,
$$

and $f \in C^{1}([0, \infty))$ satisfies some growth conditions if $p \leq n$,

$$
|f(s)| \leq C_{1} s^{q}+C_{2} \text { with } q \geq p-1 \text { and } q \leq \frac{n p-n+p}{n-p} \text { if } p<n .
$$

It is well known that all weak solutions of (1.1) correspond to critical points of the functional

$$
J=\frac{1}{p} \int_{B}|\nabla u|^{p} d x-\int_{B} F(u) d x,
$$

in the space $W_{0}^{1, p}(B)$, where $F(t)=\int_{0}^{t} f(s) d s$.

In the case $p=2$, many questions about the solutions of (1.1) in a general bounded smooth domain $\Omega$, are investigated by studying the linearized operator at the solution $u$, namely the operator $L_{u} v=\Delta v+f^{\prime}(u) v$. Thinking of $u$ as a critical

Received by the editors May 23, 2002.

2000 Mathematics Subject Classification. Primary 58E05, 35J05.

Research of the second author was supported by MIUR, project "Variational methods and Nonlinear Differential Equations". 
point of (1.2), this implies studying the second derivative of $J$, i.e. the bilinear form

$$
\left\langle J^{\prime \prime}(u) v, w\right\rangle=\int_{\Omega} \nabla v \cdot \nabla w d x-\int_{\Omega} f^{\prime}(u) v w d x
$$

for any $v, w$ in $H_{0}^{1}(\Omega)$. The operator $L_{u}$ is a compact perturbation of the Laplace operator and thus several properties of it are already well understood. In particular, the spectrum of $-L_{u}$ is given by a sequence of real numbers and only a finite number of them are negative. This implies that every critical point of $J$ has a finite Morse index which is defined as the supremum of the dimensions of the subspaces on which $J^{\prime \prime}(u)$ is negative definite. This allows us to use Morse theory in several ways to get the existence and uniqueness or multiplicity of solutions of (1.1) when $p=2$.

In the case $p \neq 2$, several difficulties arise in the study of the linearized operator of the $p$-Laplacian, and it is an important question to find an appropriate space where the linearized operator can provide qualitative properties of solutions.

In this paper, we consider the case of the ball and of positive radial solutions. Introducing some suitable weighted space of radial functions, we are able to get results on the spectrum of the linearized operator and derive information about the Morse index of solutions. We also get some estimates of the critical groups of $J$ at an isolated critical point and show that radial Mountain Pass solutions have Morse index 1 in the subspace $W_{0, r}^{1, p}(B)$ of radial functions in $W_{0}^{1, p}(B)$. We use this to prove uniqueness of solutions of (1.1) when $f$ is of the type $u^{s}+u^{q}$ and $p \geq 2$.

In the case $p>2, J$ is of class $C^{2}$ in $W_{0}^{1, p}(B)$ and we have for radial functions $v, w$ in $W_{0}^{1, p}(B)$

$$
\left\langle J^{\prime \prime}(u) v, w\right\rangle=\int_{B}(p-1)|\nabla u|^{p-2} \nabla v \cdot \nabla w d x-\int_{B} f^{\prime}(u) v w d x .
$$

A first difficulty concerning (1.4) is that it is defined in a Banach space, rather than in a Hilbert space, and this leads to several problems. One of these is that it does not seem possible to derive a good extension of Morse theory to a Banach space, in particular because of the existence of critical points either degenerate or with infinite Morse index. We refer the reader to [7, 8] and [15] for a deeper discussion of this point. Nevertheless, in [7, 8] some ways of studying the critical groups for functionals defined in Banach spaces are indicated. In particular in [8] the functional

$$
\frac{1}{p} \int_{\Omega}|\nabla u|^{p} d x+\frac{1}{2} \int_{\Omega}|\nabla u|^{2} d x-\int_{\Omega} F(u) d x
$$

is studied in $W_{0}^{1, p}(\Omega)$, for $p>1$. However, because of the presence of the term $\int_{\Omega}|\nabla u|^{2} d x$, this functional does not give rise to a degenerate operator as it happens for the $p$-Laplacian. Indeed, another major obstacle that one encounters in the study of $J^{\prime \prime}(u)$ is that it is a degenerate operator at any point where $\nabla u$ vanishes. Thus, understanding the properties of $J^{\prime \prime}(u)$, and, in particular, its spectrum, can be very difficult.

In this paper, motivated by some uniqueness problem that we discuss later, we are able to show several properties of $J^{\prime \prime}(u)$ in the case when $u$ is a radial solution of (1.1) such that

$$
|\nabla u| \neq 0 \text { for } x \neq 0 \text {. }
$$


Let us observe immediately that by Proposition 1.2.6 of [12] every positive solution of (1.1) satisfies (1.5) if $F(u) \leq 0$ at all values $u \leq u(0)$ for which $f(u)=0$.

It turns out that the space $W_{0, r}^{1, p}(B)$ of radial functions in $W_{0}^{1, p}(B)$ is not the right space to study $J^{\prime \prime}(u)$, but two other spaces come naturally into the picture, one larger than $W_{0, r}^{1, p}(B)$ and the other one smaller. To be more precise, let us observe that any solution of (1.1) satisfying (1.5) belongs to $C^{2}(\bar{B} \backslash\{0\}) \cap C^{1}(\bar{B})$, by the result of [12]. Moreover, using the l'Hopital rule, it is possible to derive the precise behaviour of $|\nabla u|$ near zero, which is exactly like $|x|^{1 /(p-1)}$. This, in light of the theory of degenerate operators of Murthy and Stampacchia [16] leads to the determination of a Hilbert space $H \supset W_{0, r}^{1, p}(B)$ given by functions $v$ such that $\int_{B}|x|^{\frac{p-2}{p-1}}|\nabla v|^{2} d x<\infty$. This space is suitable for studying the linearized operator $L_{u}$ defined by

$$
\left\langle L_{u} v, w\right\rangle=-\int_{B}(p-1)|\nabla u|^{p-2} \nabla v \cdot \nabla w d x+\int_{B} f^{\prime}(u) v w d x, \quad v, w \in H .
$$

Note that (1.6) is the same as $\left\langle J^{\prime \prime}(u) v, w\right\rangle$ whenever $v, w \in W_{0, r}^{1, p}(B)$. One important feature of $-L_{u}$ in the space $H$ is that its spectrum is given by a sequence of eigenvalues $\left\{\mu_{k}\right\}$, and only a finite number of them are negative or zero. Note that in $H, J$ is not defined, but proving directly some regularity results, we are able to show that all the eigenfunctions of $L_{u}$ are in fact in a more regular space of radial functions, namely

$$
\begin{aligned}
X= & \left\{v \in C^{2}(\bar{B} \backslash\{0\}) \cap C^{1}(\bar{B}), \text { s.t. } v=0 \text { on } \partial B, v(x)=w(|x|)\right. \\
& \text { with } \left.\left|w^{\prime}(|x|)\right| \leq c|x|^{\frac{1}{p-1}},\left|w^{\prime \prime}(|x|)\right| \leq c|x|^{\frac{2-p}{p-1}}, \forall x \in B \backslash\{0\}\right\},
\end{aligned}
$$

which is contained in the Hölder space $E=C_{0, r}^{1,1 /(p-1)}(\bar{B}), p>2$. This type of space was introduced in [18] in connection with the study of Ginzburg-Landau problems. We have already used the space $X$ in 2 for the study of uniqueness of positive solutions for $p$-Laplace equations with nonlinearities different from the ones considered in the next theorem.

All this allows us to prove that every critical point of $J$ has a finite Morse index in $W_{0, r}^{1, p}(B)$ and it is possible to estimate the critical groups of the functional $J$, and of its restriction $J_{X}$ to the space $X$, using the same approach as in 8 .

In particular, we prove that all solutions of (1.1) satisfying (1.5) which are critical points of $J$ of "mountain-pass" type have Morse index one in the spaces $X$ and $W_{0, r}^{1, p}(B)$. This information is, of course, important in itself, but it will be crucial for us to get the uniqueness result discussed later.

Finally, using again the theory of weighted Sobolev spaces but for linear operators with unbounded coefficients (as in 24]) and the space $X$ we are able to show some results also in the case $p<2$. Note that $J$ is not twice differentiable in $W_{0, r}^{1, p}(B)$ for $p<2$ but is of class $C^{2}$ if restricted to the space $X$.

All this will be explained in details and proved in sections 2 and 3 where also the precise statements of the results will be given.

The key tools in this part are to introduce the spaces $H$ and $X$. In order to extend our theory to general domains for solutions such that $|\nabla u|=0$ at isolated points, one would need to have information about the behaviour of $\nabla u$ near its zeroes. Once this would be achieved, one can adapt the space $X$ in such a way 
that the weight is required near each critical point of $u$ (see [18]) and the theory for evaluating the Morse index would follow.

The last part of the paper deals with the question of uniqueness of positive radial solutions to problem (1.1) when $p \geq 2$.

We assume that $f$ satisfies

(H1) $f \in C^{1}([0, \infty)), f(0)=0, f^{\prime}(t) \geq 0$ for $t>0$,

(H2) $p-1 \leq s \leq u f^{\prime}(u) / f(u) \leq q$ for some $s$ and $q$,

(H3) $(q-p+1)(n p F(u)-(n-p) u f(u))+p\left(u f^{\prime}(u)-q f(u)\right) u \geq 0$.

These hypotheses include nonlinearities of the type

$$
u^{s}+u^{q} \quad \text { with } \quad p-1 \leq s<q .
$$

Note that in this case $(H 3)$ is equivalent to

$$
(n-p) q \leq n p-n+p \quad \text { and } \quad(s+1-p)(p(s+1)-n(q+1-p)) \geq 0 .
$$

In particular, if $s=p-1,(H 3)$ holds for any $q$ subcritical or critical. Another way to write (1.8) is to define $\alpha=q-s$ and $\beta=s-(p-1)$; then (1.8) implies

$$
n \alpha+\beta(n-p) \leq p^{2} .
$$

If $\gamma=(n p-n+p) /(n-p)-q$, condition (1.8) reads $\gamma(n-p) \geq p \alpha$.

We first derive a nondegeneracy result:

Theorem 1.1. Let $f$ be a function satisfying $(H 1)-(H 3)$. For $p \geq 2$, any weak solution of (1.1) with Morse index at most 1 is nondegenerate.

We use in a crucial way all the information derived in section 2 about the operator $L_{u}$ in the case $p>2$. In particular, the second eigenvalue of $-L_{u}$ in $X$ is nonnegative for solutions of Morse index one and this implies that a solution of the linearized operator cannot have more than two nodal regions. Then, our proof follows the approach developed in 23] in the case $p=2$ and extended in 22 .

Let us notice that our hypotheses $(H 1)-(H 3)$ imply the existence of a Mountain Pass solution of $\Delta_{p} u+\lambda f(u)=0$ in the ball for all positive $\lambda$ if $s>p-1$ and $q<(n p-n+p) /(n-p)$. Indeed the hypotheses of [19] are satisfied. In the case $s=p-1$, and $q<(n p-n+p) /(n-p)$, one needs additionally that $f(u) / u^{p-1}$ tends to some limit $k$ to get the existence from [19] for all $\lambda \in\left(0, \lambda_{1, p} / k\right)$, where $\lambda_{1, p}$ is the first eigenvalue of the nonlinear problem $-\Delta_{p} u=\lambda|u|^{p-2} u$ with Dirichlet boundary conditions. If $\lambda>\lambda_{1, p} / k$, then there is no solution. If $q$ is critical, that is equal to $(n p-n+p) /(n-p)$, then more details on the existence of Mountain Pass solutions can be found in [3, 5, 13]. In particular, if $f$ is exactly the sum of 2 powers with $q$ critical and $s=p-1$, there is a Mountain Pass solution for all $\lambda \in\left(0, \lambda_{1, p}\right)$ if $n \geq p^{2}$, and only for $\lambda \in\left(\underline{\lambda}, \lambda_{1, p}\right)$ for $p<n<p^{2}$ and for some positive $\underline{\lambda}$.

Then we derive uniqueness from our nondegeneracy result and the existence of a Mountain Pass solution. Indeed, we prove in section 2 that Mountain Pass solutions have index 1 . Then we use a bifurcation analysis. This requires an implicit function theorem that we derive, as in [2], in the space $X$. We show that all solutions lie on a simple curve, made by solutions of Morse index one, which does not have any turning point, because of the nondegeneracy. We can prove the following uniqueness theorem. 
Theorem 1.2. Let $f$ be a function satisfying $(H 1)-(H 3)$. Assume that either

- $q<(n p-n+p) /(n-p)$ and $s>p-1$,

- or $q<(n p-n+p) /(n-p), s=p-1$ and $f(u) / u^{p-1}$ tends to some limit $k$ as $u$ tends to 0 ,

- or $q=(n p-n+p) /(n-p), f(u)=u^{s}+u^{q}$, with $s \geq p-1$ if $n \geq p^{2}$ or $s \geq p(n p+p-2 n) /(p-1)(n-p)$ if $n<p^{2}$.

If $p \geq 2$, there is at most one positive radial solution of (1.1) and it has Morse index one.

In the case $u^{q}+u^{p-1}, p<n$ and $q \leq(n p-n+p) /(n-p)$, the uniqueness part of Theorem 1.2 was already proved in [1] by another method. More general nonlinearities were considered by Erbe and Tang [11 but with hypotheses of a different kind since they treat the case $\alpha \leq 1$. Note that hypothesis (H3) implies that the function $H$ defined in [11] is negative. However our proof is also different from that of 11 .

\section{THE CASE $p>2$}

2.1. Preliminaries. Let $u$ be a weak radial solution of (1.1) satisfying (1.5). As recalled in the introduction, $u$ is a critical point of $J$ in the space $W_{0, r}^{1, p}(B)$ of radial functions in $W_{0}^{1, p}(B)$. Well-known regularity results of DiBenedetto [10] ensure that $u$ belongs to $C^{1, \alpha}(\bar{B})$ for some $\alpha>0$. Because of (1.5)), by classical regularity results, $u$ belongs in fact to $C^{2}(\bar{B} \backslash\{0\})$ (see also Ni and Serrin [17]). In radial coordinates, it means that $u$ satisfies

$$
\left\{\begin{array}{c}
\left((p-1) u^{\prime \prime}+\frac{n-1}{r} u^{\prime}\right)\left|u^{\prime}\right|^{p-2}+f(u)=0 \text { in }(0,1), \\
u>0 \text { in }(0,1), \quad u^{\prime}(0)=u(1)=0 .
\end{array}\right.
$$

Moreover, by (1.5), we have $u^{\prime}(r)<0$ in $(0,1)$.

Actually, one can derive a precise behaviour of $u$ near the origin using the l'Hopital rule (see 20]). Indeed, it follows from the equation that as $r$ tends to zero, $\left(\left|u^{\prime}\right|^{p-1} r^{n-1}\right)^{\prime} /\left(r^{n}\right)^{\prime}$ tends to a finite limit; hence $\left(\left|u^{\prime}\right|^{p-1} r^{-1}\right)$ tends to the same limit, i.e.

$$
u^{\prime}(r) r^{\frac{-1}{p-1}} \rightarrow-\left(\frac{f(u(0))}{n}\right)^{\frac{1}{p-1}} \text { as } r \rightarrow 0 .
$$

Moreover, we derive from the equation (2.1) that

$$
u^{\prime \prime}(r) r^{\frac{p-2}{p-1}} \rightarrow-\frac{1}{p-1}\left(\frac{f(u(0))}{n}\right)^{\frac{1}{p-1}} \text { as } r \rightarrow 0 .
$$

This implies that $u$ lies in the space $X$ defined in (1.7) in the introduction, which is a Banach space with the norm

$$
\|v\|_{X}=\|v\|_{C^{2}\left(\bar{B} \backslash B_{1 / 2}\right)}+\sup _{0<s<1 / 2} \frac{\left|v^{\prime}(s)\right|}{s^{\frac{1}{p-1}}}+\sup _{0<s<1 / 2} \frac{\left|v^{\prime \prime}(s)\right|}{s^{\frac{2-p}{p-1}}} .
$$

Any radial solution $u$ of (1.1) is a critical point of the functional $J$ in $W_{0, r}^{1, p}(B)$. For $p>2, J \in C^{2}\left(W_{0, r}^{1, p}(B)\right)$, and for radial critical points

$$
\left\langle J^{\prime \prime}(u) v, w\right\rangle=\left\langle A_{u} v, w\right\rangle-\int_{B} f^{\prime}(u) v w d x, \quad \forall v, w \in W_{0, r}^{1, p}(B),
$$


where

$$
\left\langle A_{u} v, w\right\rangle=(p-1) \int_{B}|\nabla u|^{p-2} \nabla v \cdot \nabla w d x .
$$

Since $W_{0, r}^{1, p}(B)$ is a Banach space which is not isomorphic to its dual space, the operator $A_{u}$ is not a Fredholm operator in $W_{0, r}^{1, p}(B)$. Moreover, $A_{u}$ is a degenerate operator at the origin where $\nabla u$ vanishes. But by (2.2), we know that $|\nabla u|$ behaves like $|x|^{1 /(p-1)}$ near the origin. This allows us to use the theory of Murthy and Stampacchia [16] to study the degenerate operator $A_{u}$ in a suitable weighted Sobolev space.

Let us set $m(x)=|x|^{(p-2) /(p-1)}$ and observe that $m \in L^{\infty}(B)$ while $1 / m \in L^{t}(B)$ for any $t<n(p-1) /(p-2)$. Since $p>2$, we can choose $t \geq n$. Then let us define the weighted space $H=H_{0, r}^{1}(m, B)$ as the closure of the space $C_{0, r}^{1}(B)$ of radial $C^{1}$ functions with compact support in $B$, with respect to the scalar product

$$
(v, w)=\int_{B}|x|^{\frac{p-2}{p-1}} \nabla v \cdot \nabla w d x
$$

The space $H$ is a Hilbert space and because of the behaviour of $|\nabla u|$ at the origin, an equivalent norm is given by

$$
\|v\|_{H}=\int_{B}|\nabla u|^{p-2}|\nabla v|^{2} d x .
$$

Hence the bilinear form

$$
\left\langle A_{u} v, w\right\rangle=(p-1) \int_{B}|\nabla u|^{p-2} \nabla v \cdot \nabla w d x, \quad v, w \in H,
$$

is coercive in $H$. Note that $W_{0, r}^{1, p}(B)$ is included in $H$ and we are denoting in the same way the operator in $W_{0, r}^{1, p}(B)$ and its extension to $H$.

The canonical inclusion of $H$ into $L_{r}^{2}(B)$, which is the space of $L^{2}$ radial functions, is completely continuous (see [16]). Hence applying the Riesz Fredholm theory for completely continuous operators in a Hilbert space, we obtain that the spectrum of $A_{u}$ is given by a sequence of positive real numbers and the corresponding system of eigenfunctions is an orthonormal system in $L_{r}^{2}(B)$. Then we consider in $H$ the extension of $J^{\prime \prime}(u)$ that we denote by $-L_{u}$ :

$$
\left\langle-L_{u} v, w\right\rangle=\left\langle A_{u} v, w\right\rangle-\left\langle f^{\prime}(u) v, w\right\rangle .
$$

Proposition 2.1. The spectrum of $-L_{u}$ in $H$ is given by a sequence of eigenvalues and only a finite number of them are nonpositive. Any eigenfunction of $-L_{u}$ in $H$ in fact belongs to $X$.

Proof. $-L_{u}$ is a compact perturbation of $A_{u}$ since the operator $K_{u}$ defined by $\left\langle K_{u} v, w\right\rangle=\left\langle f^{\prime}(u) v, w\right\rangle$ is compact from $H$ into its dual space. Hence the spectrum is given by a sequence of eigenvalues $\mu_{1}<\mu_{2} \leq \cdots$ and only a finite number of them are nonpositive. Let $v$ be a radial eigenfunction in $H$ of our linearized operator corresponding to an eigenvalue $\mu$. Hence, in radial coordinates, $v$ solves

$$
(p-1)\left(r^{n-1}\left|u^{\prime}\right|^{p-2} v^{\prime}\right)^{\prime}+r^{n-1} f^{\prime}(u) v=-\mu r^{n-1} v, \quad v(1)=0 .
$$

Moreover, testing against $C^{1}$ functions implies $\lim _{r \rightarrow 0} r^{n-1}\left|u^{\prime}\right|^{p-2} v^{\prime}=0$. Then using the l'Hopital rule and equation (2.6), we deduce, as in (2.2), that $v^{\prime}$ has the 
same behaviour as $u^{\prime}$, namely

$$
v^{\prime}(r) r^{\frac{-1}{p-1}} \rightarrow-v(0)\left(\frac{\mu+f^{\prime}(u(0))}{n(p-1)}\right)\left(\frac{f(u(0))}{n}\right)^{\frac{2-p}{p-1}} \text { as } r \rightarrow 0 .
$$

Moreover, since $u^{\prime}$ does not vanish away from 0 , it follows that $v$ is $C^{2}(\bar{B} \backslash\{0\})$. Similarly as for $u$, one can derive the behaviour of $v^{\prime \prime}$ from the equation so that $v^{\prime \prime}(r) r^{\frac{p-2}{p-1}}$ has a limit as $r$ tends to 0 . In particular $v$ is in $X$.

By [16], we can also derive that the first eigenvalue is simple and the corresponding eigenfunction does not change sign, while all the others change sign. In particular, we have

Proposition 2.2 (Courant's nodal domain theorem). Any radial eigenfunction corresponding to the $k^{\text {th }}$ eigenvalue $\mu_{k}$ has at most $k$ nodal domains. In particular, any second eigenfunction has exactly two nodal domains.

Proof. As for the case of uniformly elliptic operators, one can produce a variational characterization of the eigenvalues in the space $H$. Then, since we are considering radial functions, it is easy to see, by the uniqueness theorem for ODE's, that any eigenvalue is simple. Then the classical proof applies (see for instance [4]) and the assertion follows.

Let us define the space

$$
E=C_{0, r}^{1,1 /(p-1)}(B)
$$

which is the space of radial Hölder functions with Hölder exponent $1 /(p-1)$ and 0 boundary value. We have

Proposition 2.3. $X$ is continuously embedded in $E$.

Proof. Let $v$ be in $X$. In $\bar{B} \backslash B_{1 / 2}, v$ is $C^{2}$, hence it is Hölder continuous. We estimate $v^{\prime}$ in $B_{1 / 2}$ using radial coordinates. For any $0 \leq r_{1}<r_{0}$, we need to estimate

$$
\left|\frac{v^{\prime}\left(r_{0}\right)-v^{\prime}\left(r_{1}\right)}{\left(r_{0}-r_{1}\right)^{1 /(p-1)}}\right|
$$

in terms of $\|v\|_{X}$. If $r_{1}<r_{0} / 2$, we bound (2.8) by

$$
C \sup _{\left(r_{1}, r_{0}\right)}\left|v^{\prime}\right| r_{0}^{\frac{-1}{p-1}} \leq C\|v\|_{X} .
$$

On the other hand, if $r_{1} \geq r_{0} / 2$, we have

$$
\left|\frac{v^{\prime}\left(r_{0}\right)-v^{\prime}\left(r_{1}\right)}{\left(r_{0}-r_{1}\right)^{1 /(p-1)}}\right| \leq\left(r_{0}-r_{1}\right)^{\frac{p-2}{p-1}} \sup _{\left(r_{1}, r_{0}\right)}\left|v^{\prime \prime}\right| .
$$

We use the estimate for $v^{\prime \prime}$ given by the definition of the space $X$ which implies that

$$
\left|v^{\prime \prime}(r)\right| \leq\|v\|_{X} r^{\frac{2-p}{p-1}} .
$$

Since $r_{0} / r_{1}-1<1$, it follows that (2.8) is bounded by

$$
\|v\|_{X} r_{1}^{\frac{2-p}{p-1}}\left(r_{0}-r_{1}\right)^{\frac{p-2}{p-1}} \leq\|v\|_{X} .
$$


2.2. A first estimate of the critical groups. We want to study the behaviour of the functional $J$ near a solution $u$ of (1.1) satisfying (1.5), which we assume to be an isolated critical point of $J$ in $W_{0, r}^{1, p}(B)$. Let $J_{X}$ be the restriction of $J$ to the space $X$. Since $u$ is an isolated critical point of $J$ in $W_{0, r}^{1, p}(B)$, it is also an isolated critical point of $J_{X}$ in $X$.

Definition 2.1. Let $c=J(u)$ and let us define

$$
\begin{gathered}
J^{c}=\left\{\phi \in W_{0, r}^{1, p}(B) \text { s.t. } J(\phi) \leq c\right\}, \\
J_{X}^{c}=\left\{\phi \in X \text { s.t. } J_{X}(\phi) \leq c\right\} .
\end{gathered}
$$

The $i^{\text {th }}$ critical groups of $J$ and $J_{X}$ at $u$ are defined as

$$
C_{i}(J, u)=H^{i}\left(J^{c}, J^{c} \backslash\{u\}\right) \text { and } C_{i}\left(J_{X}, u\right)=H^{i}\left(J_{X}^{c}, J_{X}^{c} \backslash\{u\}\right)
$$

where $H^{i}(A, B)$ denotes the $i^{\text {th }}$ Alexander-Spanier cohomology group of the pair $(A, B)$ with coefficients in a field $\mathbb{K}$.

The Morse index of $u$ as a critical point of $J$ is the supremum of the dimension of the subspaces of $W_{0, r}^{1, p}(B)$ on which $J^{\prime \prime}$ is negative definite. We denote it by $m(u)$ and we denote by $m^{*}(u)$ the sum of $m(u)$ and the dimension of the kernel of $J^{\prime \prime}(u)$. Sometimes, $m^{*}(u)$ is called the large Morse index of $u$. In $X$, the same definitions hold and we denote by $m_{X}(u)$ and $m_{X}^{*}(u)$ the Morse indices of $u$ as a critical point of $J_{X}$.

In the previous section, we have studied the extension of $J^{\prime \prime}(u)$ to the Hilbert space $H$. Though the functional $J$ is not defined in the whole space $H$, it is natural to expect a relation between the number of negative eigenvalues of $-L_{u}$ in $H$ and the Morse indices of $u$. Moreover, in view of Proposition 2.1] it is natural to expect that the Morse indices are the same in $W_{0, r}^{1, p}(B)$ and in $X$.

Theorem 2.1. Let $u$ be an isolated critical point of $J$ in $W_{0, r}^{1, p}(B)$ with $u>0$ and satisfying (1.5). Then the Morse indices of $u$ are finite. More precisely,

$$
m(u)=m_{X}(u)=N\left(L_{u}\right) \quad \text { and } \quad m^{*}(u)=m_{X}^{*}(u)=N_{0}\left(L_{u}\right)
$$

where $N\left(L_{u}\right)$ and $N_{0}\left(L_{u}\right)$ are respectively the number of negative and nonpositive eigenvalues of $L_{u}$ in $H$. Moreover,

$$
C_{i}(J, u)=C_{i}\left(J_{X}, u\right)=\{0\} \quad \text { for any } \quad i \leq m_{X}(u)-1 .
$$

As in [8], the proof of (2.10) follows from the regularity of the eigenfunctions while (2.11) is a consequence of the following result of Lancellotti [14]:

Proposition 2.4 ([14]). Let $Y$ be a Banach space and $I: Y \rightarrow \mathbb{R}$ a continuous functional. Assume that there exists a subspace $V$ of $Y$ of finite dimension $m$ such that

i) for every $u$ in $Y$, the functional $I$ is of class $C^{2}$ on $u+V$ and for every $v$ in $V$, the functions $u \rightarrow\left\langle I^{\prime}(u), v\right\rangle$ and $u \rightarrow\left\langle I^{\prime \prime}(u) v, v\right\rangle$ are continuous on $Y$,

ii) $\left\langle I^{\prime \prime}(0) v, v\right\rangle<0$ for every $v$ in $V \backslash\{0\}$.

Then

$$
C_{i}(I, 0)=\{0\} \text { for every } i \leq m-1 .
$$


Proof of Theorem [2.1] By the theory explained in section 2.1, N(L $\left.L_{u}\right)$ and $N_{0}\left(L_{u}\right)$ are finite and represent respectively the maximal dimension of a subspace of $H$ on which $-L_{u}$ is negative definite and the sum of it with the kernel of $L_{u}$. Hence

$$
m_{X}(u) \leq m(u) \leq N\left(L_{u}\right) .
$$

Since $L_{u}$ is a Fredholm operator in $H$, we can consider the splitting

$$
H=H^{-} \oplus H^{0} \oplus H^{+},
$$

where $H^{-}, H^{0}$ and $H^{+}$are the spaces spanned by the eigenfunctions corresponding respectively to the negative, null and positive eigenvalues of $-L_{u}$ in $H$. In addition, we have

$$
\exists \beta>0 \text { such that }\left\langle L_{u} v, v\right\rangle \geq \beta\|v\|_{H}^{2}, \quad \forall v \in H^{+} .
$$

By Proposition 2.1 we know that each eigenfunction is in $X$. Thus, since $H^{-}$and $H^{0}$ are finite dimensional, we have that $H^{-} \oplus H^{0} \subset X \subset W_{0, r}^{1, p}(B)$, so that

$$
\begin{aligned}
& W_{0, r}^{1, p}(B)=M \oplus Z \text { where } M=H^{-} \oplus H^{0} \text { and } Z=H^{+} \cap W_{0, r}^{1, p}(B), \\
& X=M \oplus Z_{X} \text { where } Z_{X}=Z \cap X .
\end{aligned}
$$

Moreover, by (2.12), we have $\left\langle L_{u} v, v\right\rangle \geq \beta\|v\|_{H}^{2}, \forall v \in Z$, which together with (2.13) implies (2.10).

Finally (2.11) is just a consequence of Proposition 2.4 and of (2.10), taking $H^{-}$ as the finite dimensional subspace $V$ of $W_{0, r}^{1, p}(B)$ or of $X$ in the statement of the proposition.

Corollary 2.1. If $u$ is an isolated critical point of Mountain Pass type of the functional $J$ in $W_{0, r}^{1, p}(B)$, with $u>0$ and satisfying (1.5), then its Morse index in $X$ or in $W_{0, r}^{1, p}(B)$ is at most one. In other words, only the first eigenvalue of the operator $-L_{u}$ in $H$ can be negative.

Proof. Since $u$ is a Mountain Pass solution, the first critical group is nontrivial (see [6]), which implies by (2.11), that $m(u) \leq 1$.

Remark 2.1. The result of Corollary 2.1] could be generalized to critical points of other mini-max types using the fact that for this kind of points, some critical groups are nontrivial (see [6]).

2.3. Further estimates of the critical groups. In this section, we will derive other estimates for the critical groups which, together with (2.11), will provide the natural estimates usually stated in Morse theory. We will do it for the functional $J_{X}$.

Theorem 2.2. Let $u$ be an isolated critical point of $J_{X}$ in $W_{0, r}^{1, p}(B)$ with $u>0$ and satisfying (1.5). Then

$$
C_{i}\left(J_{X}, u\right)=\{0\}, \quad \text { for any } i \geq m^{*}(u)+1 .
$$

Moreover, if 0 is not an eigenvalue of $L_{u}$ in $H$, then

$$
C_{i}\left(J_{X}, u\right) \simeq \mathbb{K} \text { if } i=m(u) \text { and } C_{i}\left(J_{X}, u\right)=\{0\} \text { if } i \neq m(u) .
$$


To prove this, we follow the approach of [8] (see also [14]), i.e. we show a convexity property of $J_{X}$ near $u$ and from this, we derive a finite dimensional reduction which allows us to prove (2.14). However, as compared with [8], our proof is simpler since we work in a space of more regular functions; also the way we get the finite dimensional reduction is quite different and more direct.

Let us recall that, as defined in (2.13), $M=H^{-} \oplus H^{0}$ and $Z=H^{+} \cap W_{0, r}^{1, p}(B)$, while $Z_{X}=Z \cap X$.

Lemma 2.1. There exist $\delta_{0}$ and $C>0$ such that for any $z \in X$ with $\|z-u\|_{X}<\delta_{0}$,

$$
\left\langle J_{X}^{\prime \prime}(z) w, w\right\rangle \geq C\|w\|_{H}^{2} \quad \forall w \in Z_{X} .
$$

Moreover

$$
J_{X}(u+w)>J_{X}(u) \quad \forall w \in Z_{X} \backslash\{0\} \text { with }\|w\|_{X} \leq \delta_{0} .
$$

Proof. To prove (2.15), we argue by contradiction and assume that there are two sequences $z_{n}$ in $X$ and $w_{n}$ in $Z_{X} \backslash\{0\}$ such that $\left\|w_{n}\right\|_{H}=1,\left\|z_{n}-u\right\|_{X}$ tends to 0 and

$$
\left\langle J_{X}^{\prime \prime}\left(z_{n}\right) w_{n}, w_{n}\right\rangle \leq 1 / n .
$$

Since $w_{n}$ is bounded in $H$, it converges weakly in $H$, up to a subsequence, to a function $w$ in $H^{+}$and hence strongly in $L^{2}(B)$. By the convergence of $z_{n}$ to $u$ in $X$, we get that

$$
\frac{\left|\nabla z_{n}\right|}{|\nabla u|} \rightarrow 1 \text { uniformly in } B
$$

and hence, since $\left\|w_{n}\right\|_{H}=1=\int_{B}|\nabla u|^{p-2}\left|\nabla w_{n}\right|^{2} d x$, we obtain

$$
\int_{B}\left|\nabla z_{n}\right|^{p-2}\left|\nabla w_{n}\right|^{2} d x \rightarrow 1 \text {. }
$$

Moreover, $\int_{B} f^{\prime}\left(z_{n}\right) w_{n}^{2} d x$ tends to $\int_{B} f^{\prime}(u) w^{2} d x$. This implies

$$
0 \geq \lim \left\langle J_{X}^{\prime \prime}\left(z_{n}\right) w_{n}, w_{n}\right\rangle \geq 1+\int_{B} f^{\prime}(u) w^{2} d x
$$

from which we deduce that $w$ cannot be identically zero. Moreover, from (2.12), (2.17) and the lower semicontinuity of the norm in $H$, we get

$$
0 \geq\left\langle J_{X}^{\prime \prime}(u) w, w\right\rangle \geq \beta\|w\|_{H}^{2},
$$

which is a contradiction. The inequality (2.16) follows from (2.15) since we have

$$
J_{X}(u+w)-J_{X}(u)=\frac{1}{2}\left\langle J_{X}^{\prime \prime}(z) w, w\right\rangle \geq C\|w\|_{H}^{2}>0
$$

for any $w$ in $Z_{X} \backslash\{0\}$ with $\|w\|_{X}<\delta_{0}$ and some $z$ in $X$ with $\|z-u\|_{X}<\delta_{0}$.

Lemma 2.2. There exist $\rho, \delta \in\left(0, \delta_{0}\right)$ such that for any $v$ in $M$ with $\|v\|_{X} \leq \rho$, there exists exactly one point $\bar{w}=\psi(v)$ in $Z_{X} \cap D_{\delta}$, where $D_{\delta}=\left\{w \in X,\|w\|_{X}<\right.$ $\delta\}$, satisfying

$$
\left\langle J_{X}^{\prime}(u+v+\psi(v)), z\right\rangle=0 \quad \forall z \in Z_{X} .
$$

Moreover, $\psi(v)$ is the only minimum of the function $G(w)=J(u+v+w)$ in $Z_{X} \cap D_{\delta}$. Finally, the map $\psi: M \cap \bar{D}_{\rho} \rightarrow Z_{X} \cap D_{\delta}$ is of class $C^{1}$. 
To construct the map $\psi$, we will use the implicit function theorem. Let us consider the map $B$ defined in $M \times Z_{X}$ by

$$
B(v, w)=\frac{\partial J_{X}}{\partial w}(u+v+w)
$$

that is, $B$ is the derivative of $J_{X}$ with respect to $w \in Z_{X}$. One can derive that $B$ takes its values in $L^{\infty} \cap C^{0}(0,1]=\mathcal{E}_{0}^{0}$. We have that $B(0,0)=\left(\partial J_{X} / \partial w\right)(u)=0$. We would like to show that $(\partial B / \partial w)(0,0)$ is an isomorphism between $Z_{X}$ and $\mathcal{E}_{0}^{0}$ to be able to use the implicit function theorem. We have

$$
\frac{\partial B}{\partial w}(0,0)=\frac{\partial^{2} J_{X}}{\partial w^{2}}(u)
$$

This is represented by $-L_{u}$, taken against test functions in $Z_{X}$. Because of the coercivity (2.12), $L_{u}$ is injective in $H^{+}$, hence in $Z_{X}$. Now we need to prove surjectivity. For any $\phi$ in $\mathcal{E}_{0}^{0}$, by the coercivity and Lax-Milgram theorem, there exists $v$ in $H^{+}$such that $L_{u} v=\phi$ in $H^{+}$, that is

$$
\int_{B}|\nabla u|^{p-2} \nabla v \cdot \nabla w d x-\int_{B} f^{\prime}(u) v w d x=\int_{B} \phi w d x \quad \forall w \in H^{+} .
$$

Let $e_{i}(r)$ be an orthonormal basis of $M$, for any $h$ in $H$; then $h-\sum_{i}\left(\int h e_{i}\right) e_{i}$ is in $H^{+}$. Hence using it as a test function, we have that $v$ satisfies in radial coordinates

$$
(p-1)\left(r^{n-1}\left|u^{\prime}\right|^{p-2} v^{\prime}\right)^{\prime}+r^{n-1}\left(f^{\prime}(u) v+l(r, u, v)+\phi\right)=0 \text { in }(0,1)
$$

where

$$
l(r, u, v)=\sum_{i} e_{i}(r) \int_{B}\left(|\nabla u|^{p-2} \nabla v \cdot \nabla e_{i}-f^{\prime}(u) v e_{i}-\phi e_{i}\right) d x .
$$

Since $\phi$ is in $\mathcal{E}_{0}^{0}$, we have that $v$ is indeed in $X$ and because it is also in $H^{+}$, it is therefore in $Z_{X}$.

Then we can apply the implicit function theorem to $B$ in a neighborhood of $(0,0)$, to get the existence of $\rho, \delta>0$ and a continuous map

$$
\psi: M \cap \bar{D}_{\rho} \rightarrow \bar{w} \in Z_{X} \cap D_{\delta}
$$

such that for any $v$ in $M \cap D_{\rho}, \psi(v)$ is the only point in $Z_{X} \cap D_{\delta}$ satisfying (2.18). Moreover, $\psi$ is of class $C^{1}$ because $J_{X}^{\prime}$ is also continuously differentiable with respect to $v$. Finally, assuming without loss of generality that $\rho, \delta$ are smaller than $\delta_{0}$, we get from the strict convexity of $J_{X}$, proved in (2.15) , that $\psi(v)$ is the only minimum point of $G$ in $Z_{X} \cap D_{\delta}$.

Proof of Theorem 2.2. Let us define the functional $\Phi(v)=J(u+v+\psi(v))$ for any $v \in M \cap \bar{D}_{\rho}$. From the previous lemma, $\Phi$ is continuous and denoting by $\Phi^{c}$ its sublevels, that is

$$
\Phi^{c}=\left\{v \in M \cap \bar{D}_{\rho} \text { s.t. } \Phi(v) \leq c\right\},
$$

we have that the pair $\left(\Phi^{c}, \Phi^{c} \backslash\{0\}\right)$ is homeomorphic to $\left(\tilde{J}_{X}^{c}, \tilde{J}_{X}^{c} \backslash\{u\}\right)$ where $\tilde{J}_{X}$ is the restriction of $J_{X}$ to the set

$$
Y=\left\{u+v+\psi(v), v \in M \cap \bar{D}_{\rho}\right\} .
$$

Therefore, we have

$$
C_{i}\left(\tilde{J}_{X}, u\right)=C_{i}(\Phi, 0) \quad \text { for any } i
$$


On the other hand, the pair $\left(\tilde{J}_{X}^{c}, \tilde{J}_{X}^{c} \backslash\{u\}\right)$ is a deformation retract of the pair $\left(J_{X}^{c} \cap U, J_{X}^{c} \cap U \backslash\{u\}\right)$, where $U$ is a neighborhood of $u$ in $X$ given by

$$
U=u+\left(M \cap \bar{D}_{\rho}\right)+\left(Z_{X} \cap \bar{D}_{\delta}\right) .
$$

This can be seen by considering the homotopy map

$$
\gamma(t, u+v+w)=u+v+w+t(\psi(v)-w)
$$

which sends any point in $U$ to the point $u+v+\psi(v)$. Note that along the homotopy, we have that all points $g=u+v+w \in J_{X}^{c} \cap U$ remain in the subset $J_{X}^{c}$ because of (2.16). Thus,

$$
C_{i}\left(J_{X}, u\right)=H^{i}\left(\tilde{J}_{X}^{c}, \tilde{J}_{X}^{c} \backslash\{u\}\right) \simeq H^{i}\left(J_{X}^{c} \cap U, J_{X}^{c} \cap U \backslash\{u\}\right),
$$

and by the excision property, the last cohomology group is just $C_{i}\left(J_{X}, u\right)$. Therefore, by (2.22)-2.23), to prove the assertion, we just have to estimate the critical groups of the function $\Phi$ which is defined in a finite dimensional space. Then (2.14) is trivial because $C_{i}(\Phi, 0)$ must be zero for any $i>\operatorname{dim} M$ which is $m^{*}(u)$. If we assume in addition that 0 is not an eigenvalue of $L_{u}$ in $H$, we have that $M=H^{-}$and hence $u$ is a local maximum of $J$ in $M$. This implies that 0 is a nondegenerate local maximum of $\Phi$ in the finite dimensional space $M$, so that $C_{i}(\Phi, 0)$ is isomorphic to the field $\mathbb{K}$ if $i=\operatorname{dim} M=m(u)$ while $C_{i}(\Phi, 0)=\{0\}$ if $i \neq m(u)$.

\section{THE CASE $p<2$}

Let $u$ be a weak radial solution of (1.1) satisfying (1.5). Arguing exactly as in the beginning of section 2, we have that $u$ belongs to the space $X$ defined in the introduction.

Note that the functional $J$ is not twice differentiable in $W_{0, r}^{1, p}(B)$, but we can prove that it is $C^{2}$ in $X$.

Proposition 3.1. $J_{X} \in C^{2}(X)$ and we have (1.4) for any $v, w$ in $X$.

Proof. When one computes $\left\langle J_{X}^{\prime}(u+\varepsilon v), w\right\rangle-\left\langle J_{X}^{\prime}(u), w\right\rangle-\varepsilon\left\langle J_{X}^{\prime \prime}(u) v, w\right\rangle$, one has to estimate integrals of the type

$$
\int_{B}|\nabla u|^{p-2} \frac{|\nabla v|^{2}}{|\nabla u|^{2}} \nabla u \cdot \nabla w d x .
$$

Using the precise behaviour of $\nabla u$ near the origin, this is less than

$$
C\|v\|_{X}^{2}\|w\|_{X},
$$

where $C$ depends on $u$.

Note that if one wants to get $C^{2}$ differentiability of $J$ in the space $C^{2}(\bar{B})$, this is true for $p$ bigger than $(n+6) /(n+4)$.

Then we can define the Morse index of $u$ as a critical point of $J_{X}$ as the supremum of the dimensions of the subspaces of $X$ on which $J_{X}^{\prime \prime}(u)$ is negative definite. We denote it by $m(u)$ and we denote by $m^{*}(u)$ the sum of $m(u)$ and the dimension of the kernel of $J_{X}^{\prime \prime}(u)$.

As for the case $p>2$, we would like to study the operator $J_{X}^{\prime \prime}(u)$ in a suitable weighted Hilbert space. To this aim, we consider the function $m(x)=|x|^{(p-2) /(p-1)}$ and observe that $m \in L^{1}(B)$ if $p>(n+2) /(n+1)$ while $1 / m \in L^{\infty}(B)$. Then for $p>(n+2) /(n+1)$, we define the weighted space $H=H_{0, r}^{1}(m, B)$ as the closure of 
the space $C_{0, r}^{1}(B)$ of radial $C^{1}$ functions with compact support in $B$, with respect to the scalar product

$$
(v, w)=\int_{B}|x|^{\frac{p-2}{p-1}} \nabla v \cdot \nabla w d x .
$$

As shown in 24, $H$ is a Hilbert space and by the behaviour of $|\nabla u|$ at the origin, an equivalent norm is given by

$$
\|v\|_{H}=\int_{B}|\nabla u|^{p-2}|\nabla v|^{2} d x .
$$

Hence, we have that the bilinear form

$$
\left\langle A_{u} v, w\right\rangle=(p-1) \int_{B}|\nabla u|^{p-2} \nabla v \cdot \nabla w d x
$$

is coercive in $H$. Note that $X$ is included in $H$, which is included in $W_{0, r}^{1, p}(B)$. It follows from [24] that the canonical inclusion of $H$ into $L_{r}^{2}(B)$ is completely continuous. Hence, the spectrum of $A_{u}$ is given by a sequence of positive real numbers and the corresponding system of eigenfunctions is an orthonormal system in $L^{2}(B)$. We can consider in $H$ the operator $L_{u}$ as before,

$$
\left\langle-L_{u} v, w\right\rangle=\left\langle A_{u} v, w\right\rangle-\left\langle f^{\prime}(u) v, w\right\rangle .
$$

In the case $p \leq(n+2) /(n+1)$, we cannot use the theory of [24], but we use the radial coordinates and define $m(x)=|x|^{\beta}$, where $\beta=n-1+\frac{p-2}{p-1}$. If $p \leq(n+2) /(n+1)$, then $\beta<0$. This is going to provide directly regularity on solutions. Let $\Gamma_{0, r}$ be the subspace of $C_{0, r}^{1}(B)$ where the scalar product

$$
(v, w)=\int_{0}^{1} m(x) v^{\prime} w^{\prime} d x
$$

is finite. Then $H$ is the closure of the space $\Gamma_{0, r}$ with respect to this scalar product. Obviously, $H$ is a Hilbert space and an equivalent norm is given by (3.2).

Lemma 3.1. If $p \leq(n+2) /(n+1), H$ is compactly embedded in $L^{2}(0,1)$.

Proof. The key identity is

$$
|v(s)-v(t)|^{2}=\left|\int_{t}^{s} v^{\prime}(x) d x\right|^{2} \leq\left(\int_{t}^{s} x^{\beta} v^{\prime 2}(x) d x\right)\left(\frac{s^{-\beta / 2+1}-t^{-\beta / 2+1}}{-\beta / 2+1}\right) .
$$

In particular, we deduce that any function $v$ in $H$ is in $L^{\infty}$ with

$$
\|v\|_{\infty} \leq \frac{1}{-\beta / 2+1}\|v\|_{H}^{1 / 2} .
$$

Moreover, $v$ is continuous, bounded near the origin and the unit ball of $H$ is uniformly equicontinuous since

$$
|v(s)-v(t)| \leq\|v\|_{H}^{1 / 2}|t-s|^{1 / 2}
$$

so by Ascoli's theorem, the injection of $H$ into $C^{0}(0,1)$ is compact. This implies also that $f^{\prime}(u) v$ is a compact perturbation of $A_{u}$.

Proposition 3.2. For $p<2$, the spectrum of $-L_{u}$ in $H$ is given by a sequence of eigenvalues and only a finite number of them are nonpositive. Any eigenfunction of $-L_{u}$ in $H$ in fact belongs to $X$. 
Proof. Since $-L_{u}$ is a compact perturbation of $A_{u}$, its spectrum is given by a sequence of real eigenvalues and only a finite number of them are nonpositive. Let $v$ be a radial eigenfunction in $H$. We have that $v$ is in $L^{\infty}(B)$. Moreover, $v$ is a solution of (2.6) and since $v$ is a weak solution in the whole ball $B$, we have that $r^{n-1}\left|u^{\prime}\right|^{p-2} v^{\prime}$ tends to 0 as $r$ tends to 0 . Then using the l'Hopital rule and equation (2.6) as in section 2.1, we derive the behaviour of $v^{\prime}$ and $v^{\prime \prime}$ near 0 and get that $v$ is in $X$.

Now let us assume that $u$ is an isolated critical point of $J$ in $W_{0, r}^{1, p}(B)$. Then $u$ is also an isolated critical point of $J_{X}$ in $X$. As for the case $p>2$, we have a relation between the Morse indices of $u$ and the number of negative eigenvalues of $-L_{u}$ in $H$. Moreover, we can get an estimate of the critical groups $C_{i}\left(J_{X}, u\right)$ defined in section 2 .

Theorem 3.1. Let $p<2$ and let $u$ be an isolated critical point of $J$ in $W_{0, r}^{1, p}(B)$. Then the Morse indices of $u$ are finite. More precisely,

$$
m_{X}(u)=N\left(L_{u}\right) \quad \text { and } \quad m_{X}^{*}(u)=N_{0}\left(L_{u}\right)
$$

where $N\left(L_{u}\right)$ and $N_{0}\left(L_{u}\right)$ are respectively the number of negative and nonpositive eigenvalues of $-L_{u}$ in $H$. Moreover,

$$
C_{i}\left(J_{X}, u\right)=\{0\} \text { for any } i \leq m_{X}(u)-1 .
$$

Proof. The proof is almost the same as that of Theorem 2.1. Since there are a few changes to make, we sketch it for the reader's convenience. Since $L_{u}$ is a Fredholm operator in $H$, we can consider the splitting

$$
H=H^{-} \oplus H^{0} \oplus H^{+},
$$

where $H^{-}, H^{0}$ and $H^{+}$have the same meaning as in the proof of Theorem 2.1. In particular, we have

$$
\exists \beta \text { such that }\left\langle L_{u} v, v\right\rangle \geq \beta\|v\|_{H}^{2}, \quad \forall v \in H^{+} .
$$

By Proposition 3.2, we know that each eigenfunction is in $X$, hence $H^{-} \oplus H^{0} \subset X$ and we have the splitting

$$
X=H^{-} \oplus H^{0} \oplus\left(H^{+} \cap X\right) .
$$

By this and (3.6), we get (3.4). Finally (3.5) is a consequence of Proposition 2.4 and (3.2), taking $H^{-}$as the finite dimensional subspace of $X$ in $W_{0, r}^{1, p}(B)$ in the statement of Proposition 2.4.

The other estimates of the critical groups can be obtained exactly in the same way as for the case $p>2$. Therefore we have

Theorem 3.2. Let $u$ be an isolated critical point of $J_{X}$ in $W_{0, r}^{1, p}(B)$ with $u>0$ and satisfying (1.5). Then

$$
C_{i}\left(J_{X}, u\right)=\{0\}, \quad \text { for any } i \geq m^{*}(u)+1 .
$$

Moreover, if 0 is not an eigenvalue of $L_{u}$ in $H$, then

$$
C_{i}\left(J_{X}, u\right) \simeq \mathbb{K} \text { if } i=m(u) \text { and } C_{i}\left(J_{X}, u\right)=\{0\} \text { if } i \neq m(u) .
$$




\section{Nondegeneracy AND UNiquenEss}

4.1. Proof of Theorem 1.1. Let us assume that $f$ satisfies $(H 1)-(H 3)$ and $v$ is a solution of $L_{u} v=0$ in $X$. We are going to prove that $v$ has at least three nodal regions in $B$. This and Proposition 2.2 imply that the eigenvalue which is zero has to be at least the $3 \mathrm{rd}$ one, providing a contradiction with the fact that the solution $u$ has Morse index at most 1 .

Thus let $w=v-u$; then $L_{u} w=-L_{u} u \leq 0$ by (H2). Let us notice that if we multiply the equation for $u$ by $v$ and the equation for $v$ by $u$, and integrate, we get

$$
\int_{0}^{1} r^{n-1}\left(u f^{\prime}(u)-(p-1) f(u)\right) v d r=0
$$

In $(0,1), u$ is $C^{\infty}$, so we can differentiate (2.1) with respect to $r$ to compute $L_{u}\left(r u^{\prime}\right)$ and find that $L_{u}\left(r u^{\prime}\right)=-p f(u)$. If we multiply the equation $L_{u}\left(r u^{\prime}\right)=-p f(u)$ by $v$ and the equation $L_{u} v=0$ by $r u^{\prime}$, we get

$$
p \int_{0}^{1} r^{n-1} f(u) v d r=(p-1)\left|u^{\prime}(1)\right|^{p-2} v^{\prime}(1) u^{\prime}(1) .
$$

Finally the Pohozaev identity rewrites

$$
\int_{0}^{1} r^{n-1}\left(n F(u)-\frac{n-p}{p} u f(u)\right) d r=\frac{p-1}{p}\left|u^{\prime}(1)\right|^{p-2} u^{\prime}(1)^{2} .
$$

By (4.1), we have $\int_{0}^{1} r^{n-1}\left(u f^{\prime}(u)-q f(u)\right) v d r \neq 0$ and since $v$ is always defined up to multiplication by a constant, we choose the solution $v$ such that

$$
\int_{0}^{1} r^{n-1}\left(u f^{\prime}(u)-q f(u)\right) v d r=\int_{0}^{1} r^{n-1}\left(u f^{\prime}(u)-q f(u)\right) u d r
$$

This implies

$$
\int_{0}^{1}\left(u f^{\prime}(u)-q f(u)\right) w r^{n-1} d r=0
$$

Using (4.1) and (4.2), we derive

$$
\begin{aligned}
& \int_{0}^{1} r^{n-1}\left(u f^{\prime}(u)-q f(u)\right) v d r=(p-1-q) \int_{0}^{1} r^{n-1} f(u) v d r \\
= & \frac{(p-1-q)}{p}(p-1)\left|u^{\prime}(1)\right|^{p-2} v^{\prime}(1) u^{\prime}(1) .
\end{aligned}
$$

By (4.4), we have that the last term is equal to $\int_{0}^{1} r^{n-1}\left(u f^{\prime}(u)-q f(u)\right) u d r$. By $(H 2)$ it is negative, and since by the Hopf Lemma $u^{\prime}(1)<0$, we find $v^{\prime}(1)<0$. 
Now,

$$
\begin{aligned}
& \frac{(p-1-q)}{p}(p-1)\left|u^{\prime}(1)\right|^{p-2} w^{\prime}(1) u^{\prime}(1) \\
= & \frac{(p-1-q)}{p}(p-1)\left|u^{\prime}(1)\right|^{p-2} v^{\prime}(1) u^{\prime}(1)-\frac{(p-1-q)}{p}(p-1)\left|u^{\prime}(1)\right|^{p} \\
= & \int_{0}^{1} r^{n-1}\left(\left(u f^{\prime}(u)-q f(u)\right) v-(p-1-q)\left(n F(u)-\frac{n-p}{p} u f(u)\right)\right) d r \\
= & \int_{0}^{1} r^{n-1}\left(\left(u f^{\prime}(u)-q f(u)\right) u+(q+1-p)\left(n F(u)-\frac{n-p}{p} u f(u)\right)\right) d r \\
\geq & 0 .
\end{aligned}
$$

The second inequality is obtained using the Pohozaev identity while the last integral is positive by $(H 3)$. Hence, we derive that $w^{\prime}(1)>0$. Moreover, since $\int_{0}^{1} r^{n-1}\left(u f^{\prime}(u)-q f(u)\right) w d r=0$, we have by $(H 2)$ that $w$ changes sign. Near the boundary, $w<0$, since $w^{\prime}(1)>0$. Let $D$ be the connected component of the set $\{w \neq 0\}$ near the boundary where $w<0$. In $D$, we have $w<0$ and $L_{u} w \leq 0$. Moreover we claim that $v$ has to change sign in $D$. Indeed, if not, since $L_{u} v=0$ and $v>0$ on $\partial D \cap B$, the weak version of the Maximum Principle holds since $L_{u}$ is uniformly elliptic in $D$. It implies that $w \geq 0$, which is not the case. So $v$ changes sign in $D$, but $v>0$ near the boundary since $v^{\prime}(1)<0$ and $v>0$ when $w=0$. Hence in $D, v$ has to change sign at least twice, which proves that zero cannot be the first or second eigenvalue of $L_{u}$.

4.2. Proof of Theorem 1.2. We consider problem (1.1) with a nonlinearity $\lambda f(u)$ instead of $f(u)$, where $\lambda$ is a positive free parameter:

$$
\left\{\begin{array}{l}
\left((p-1) u^{\prime \prime}+\frac{n-1}{r} u^{\prime}\right)\left|u^{\prime}\right|^{p-2}+\lambda f(u)=0 \\
u>0, \text { in }(0,1), \quad u^{\prime}(0)=u(1)=0 .
\end{array}\right.
$$

We want to describe the bifurcation diagram of solutions, that is $d=u(0)$ vs $\lambda$ in $X$.

Let us recall from 2] (Proposition 4.1) that as soon as we have proved that $L_{u}$ is injective, it implies that $L_{u}$ is an isomorphism from $X$ into $\mathcal{E}_{0}^{0}=L^{\infty} \cap C_{0}^{0}(\bar{B} \backslash\{0\})$. Indeed the surjectivity can be obtained by solving the following equation with a fixed point argument:

$$
v^{\prime}(r)=\frac{1}{r^{n-1}\left|u^{\prime}\right|^{p-2}} \int_{0}^{r} t^{n-1}\left(-f^{\prime}(u) v(t)+g(t)\right) d t,
$$

for $g \in \mathcal{E}_{0}^{0}$, with $v(0)=1$. Then we also check that the boundary condition $v(1)=0$ can be satisfied by adding a proper function $\phi$ satisfying the initial value problem $L_{u} \phi=0$ with $\phi(0)=0$ (see Proposition 4.1 of [2]).

The fact that our linearized operator is an isomorphism allows us to use the implicit function theorem in $X$ and deduce that around any solution $\left(\lambda, u_{\lambda}\right)$, there is a unique branch of solutions. Note that rescaling the equation (4.7) on the ball of radius $\lambda^{1 / p}$, problem (4.7) becomes like (2.1), but in a ball of radius $\lambda^{1 / p}$. The uniqueness theorem for initial value problems for ODE's (see [12]) yields that for any $d$, there is at most one $\lambda$ for which there is a solution of (4.7) with $u(0)=d$. In particular, if we construct a branch of solutions for which $u(0)$ goes from 0 to $\infty$, then the uniqueness for ODE's implies that there is no other branch. 
We need to start for some $\lambda$ from a Mountain Pass solution. Our hypotheses imply that this solution exists. Indeed, if $s>p-1$ and $q<(n p-n+p) /(n-p)$, there exists a Mountain Pass solution for all positive $\lambda$ from [19]. If $s=p-$ 1 and $q<(n p-n+p) /(n-p)$, our hypotheses imply the existence from [19] for all $\lambda \in\left(0, \lambda_{1, p} / k\right)$ where $\lambda_{1, p}$ is the first eigenvalue of the nonlinear problem $-\Delta_{p} u=\lambda|u|^{p-2} u$ with Dirichlet boundary conditions. If $\lambda>\lambda_{1, p} / k$, then there is no solution. If $q$ is critical, our hypotheses and the results of [3, 5, 13, imply that there is a Mountain Pass solution for all $\lambda \in\left(0, \lambda_{1, p}\right)$ if $n \geq p^{2}$ and only for $\lambda \in\left(\underline{\lambda}, \lambda_{1, p}\right)$ if $p<n<p^{2}$.

So let us start from some $\lambda_{0}$ for which there exists a Mountain Pass solution $u_{\lambda_{0}}$. Then, since the Morse Index of the solution is 1 by Theorem 1.1, it implies, respectively, that the first and second eigenvalues of the linearized operator satisfy $\mu_{1}<0$ and $\mu_{2}>0$. Using the implicit function theorem near this solution, we derive that locally there is a unique branch of solutions with the same property $\mu_{1}\left(L_{u}\right)<0$ and $\mu_{2}\left(L_{u}\right)>0$. Let us define $\underline{\lambda}$ and $\bar{\lambda}$ as the infimum and supremum of $\lambda$ on this branch.

We claim that as $\lambda$ tends to $\underline{\lambda}$ or $\bar{\lambda}, u_{\lambda}(0)$ has to go to 0 or infinity. If $\underline{\lambda}$ or $\bar{\lambda}$ is positive and finite, and if $u_{\lambda}(\overline{0})$ stays bounded, then we can find a solution for $\lambda=\underline{\lambda}$ or $\bar{\lambda}$ with either the first eigenvalue $\mu_{1}$ or the second eigenvalue $\mu_{2}$ equal to zero. This is impossible by our nondegeneracy result of Theorem 1.1 Thus the branch can be continued using the implicit function theorem and this provides a contradiction with the definition of $\underline{\lambda}$ and $\bar{\lambda}$. If $\underline{\lambda}=0$, then $u_{\lambda}(0)$ has to go to 0 or $\infty$, because the only solution for $\lambda=0$ is $u=0$. If $\bar{\lambda}=\infty$, we claim that $u_{\lambda}(0)$ has to go to 0 . The case $\bar{\lambda}=\infty$ can only happen if $s>p-1$ and $q$ is subcritical in $(H 2)$. Then, $u_{\lambda}(0)$ has to go to 0 : it cannot go to a positive finite limit because 11] proves that every solution of the linearized problem is a crossing solution and it cannot go to infinity, because rescaling the solutions and passing to the limit, we would have a solution in $\mathbb{R}^{n}$ of $\Delta_{p} u+u^{s} \leq 0$ with $s$ subcritical, which contradicts the result of Serrin and Zou [21]. Therefore, if $\bar{\lambda}=\infty, u_{\lambda}(0)$ tends to 0 as $\lambda$ tends to $\bar{\lambda}$.

Next we claim that by the uniqueness for ODE's, the limit of $u_{\lambda}(0)$ cannot be the same as $\lambda$ tends to $\underline{\lambda}$ or $\bar{\lambda}$. So the branch describes all values of $u_{\lambda}(0)$ between 0 and $\infty$ and there is no other branch.

\section{ACKNOWLEDGEMENTS}

It is a pleasure to acknowledge some interesting discussions with E.N. Dancer, K.C. Chang and S. Cingolani.

\section{REFERENCES}

[1] A.Adimurthi \& S.L.Yadava, An elementary proof of the uniqueness of positive radial solutions of a quasilinear Dirichlet problem. Arch. Rational Mech. Anal. 127 (1994), no. 3, 219-229. MR 95g:35015

[2] A.Aftalion \& F.Pacella, Uniqueness and nondegeneracy for some nonlinear elliptic problems in a ball. (2003) To appear in Journal Diff. Equ.

[3] A.Ambrosetti, J.García Azorero \& I.Peral, Multiplicity results for some nonlinear elliptic equations. J. Funct. Anal. 137 (1996), no. 1, 219-242. MR 97b:35059

[4] G.Alessandrini, On Courant's nodal domain theorem. Forum Math. 10 (1998), no. 5, 521-532. MR 99g:35091

[5] G.Arioli \& F.Gazzola, Some results on p-Laplace equations with a critical growth term. Differential Integral Equations 11 (1998), no. 2, 311-326. MR 2001b:35128 
[6] K.C.Chang, Infinite dimensional Morse theory and multiple solutions problems, Birkhäuser, Boston, Basel, Berlin (1993). MR 94e:58023

[7] K.C.Chang, Infinite dimensional Morse theory and its applications. Séminaire de Mathématiques Supérieures [Seminar on Higher Mathematics], 97. Presses de l'Université de Montréal, Montreal, QC, 1985. MR 87m:58032

[8] S.Cingolani \& G.Vannella, Critical groups computations on a class of Sobolev Banach spaces via Morse index. Ann. Inst. H. Poincaré Anal. Non Linéaire 20 (2003), no. 2, 271-292.

[9] R.Courant \& D.Hilbert, Methods of mathematical physics. Vol. I. Interscience Publishers, Inc., New York, N.Y., 1953. MR 16:426a

[10] E.DiBenedetto, $C^{1+\alpha}$ local regularity of weak solutions of degenerate elliptic equations. Nonlinear Anal. 7 (1983), no. 8, 827-850. MR 85d:35037

[11] L.Erbe \& M.Tang, Uniqueness theorems for positive radial solutions of quasilinear elliptic equations in a ball. J. Differential Equations 138 (1997), no. 2, 351-379. MR 98k:35069

[12] B.Franchi, E.Lanconelli \& J.Serrin, Existence and uniqueness of nonnegative solutions of quasilinear equations in $\mathbb{R}^{n}$. Adv. Math. 118 (1996), no. 2, 177-243. MR 97d:35056

[13] J.García Azorero \& I.Peral Alonso, Multiplicity of solutions for elliptic problems with critical exponent or with a nonsymmetric term. Trans. Amer. Math. Soc. 323 (1991), no. 2, 877-895. MR 91g:35108

[14] S.Lancellotti, Morse index estimates for continuous functionals associated with quasilinear elliptic equations. Adv. Diff. Equ. 7 (2002), 99-128. MR 2002j:58019

[15] F.Mercuri \& G.Palmieri, Problems in extending Morse theory to Banach spaces. Boll. UMI 12 (1975) 397-401. MR 53:9287

[16] M.K.V.Murthy \& G.Stampacchia, Boundary value problems for some degenerate-elliptic operators. Ann. Mat. Pura Appl. 80 (1968) 1-122. MR 40:3069

[17] W.M.Ni \& J.Serrin, Existence and nonexistence theorems for ground states for quasilinear partial differential equations. The anomalous case. Rome, Accad. Naz. dei Lincei, Attei dei Connengni 77 (1986), 231-257.

[18] F.Pacard \& T.Rivière, Linear and nonlinear aspects of vortices. The Ginzburg-Landau model. Progress in Nonlinear Differential Equations and their Applications, 39 Birkhäuser Boston, Inc., Boston, MA, 2000. MR 2001k:35066

[19] P.H.Rabinowitz, Minimax methods in critical point theory with applications to differential equations. CBMS Regional Conference Series in Mathematics, 65. Published for the Conference Board of the Mathematical Sciences, Washington, DC; by the American Mathematical Society, Providence, RI, 1986. MR 87j:58024

[20] J.Serrin \& M.Tang, Uniqueness of ground states for quasilinear elliptic equations. Indiana Univ. Math. J. 49 (2000), no. 3, 897-923. MR 2002d:35072

[21] J.Serrin \& H.Zou, Cauchy-Liouville and universal boundedness theorems for quasilinear ellptic equations. To appear, (2002).

[22] J.Shi, Exact multiplicity of positive solutions to superlinear problems. (2001) Preprint.

[23] P.N.Srikanth, Uniqueness of solutions of nonlinear Dirichlet problems. Differential Integral Equations 6 (1993), no. 3, 663-670. MR 94b:35122

[24] N.S.Trudinger, Linear elliptic operators with measurable coefficients. Ann. Scuola Norm. Pisa 27 (1973) 265-308. MR 51:6113

Laboratoire Jacques-Louis Lions, B.C. 187, Université Paris 6, 175 Rue du Chevaleret, 75013 PARIS, FRANCE

E-mail address: aftalion@ann.jussieu.fr

Dipartimento di Matematica, Università di Roma "La Sapienza", P.le A. Moro 2, 00185 ROMA, ITALY

E-mail address: pacella@mat.uniroma1.it 